\title{
Restriction Of Movement
}

Restriction of Movement (ROM) is one tool that may be considered by the Commander during an operation in order to control the spread of a contagious disease amongst personnel. It aims to control the spread of the disease by restricting contact between healthy groups of personnel and those who have, or are suspected of having, contracted the disease. ROM may also be necessary to reduce the risk that a contagious disease is transferred back to the home base.

The Commander considers the operational situation, available evidence and medical advice in assessing the likely extent of exposure and infection, in order to generate options for controlling the disease event. These might include individual and population approaches to disease control, including ROM. A Commander will need to consider ROM following evidence or suspicion (from BW sensors or health surveillance) of disease exposure to personnel.

Where ROM is implemented, it should, as far as possible, involve autonomous units that preserve the chain of command. Clear, precise and timely communication of all aspects of ROM to affected personnel is critical. Until a definitive diagnosis/ identification can be made, the Commander should assume that personnel have been exposed to, and infected by, a contagious disease and take all possible steps balanced against wider operational imperatives, to limit the spread of that disease. ROM will not be necessary for diseases that are not contagious, and may not be necessary for diseases against which physical protection, or available medical countermeasures, provide an adequate level of protection.

If $\mathrm{ROM}$ is used at any stage during an operation, it will probably have a significant impact on operational effectiveness and will restrict the use of affected force elements and their fighting effectiveness. In order to achieve a military objective limited spread of an infectious disease may be permitted.

ROM may not be enforceable on local civilians, therefore, it may be necessary to segregate forces from local populations. Security of ROM boundaries is paramount with clear guidance on handling potential breaches in either direction.
It is imperative to ensure that any contagious disease is not spread back to the home base and therefore personnel returning home from a theatre of operation where a contagious disease is confirmed, or suspected, must undergo rigorous follow-up health surveillance. Special procedures may need to be implemented for personnel and assets that cycle in and out of theatre with high frequencies, for example strategic lift aircrew.

The criteria for ending ROM should be determined before implementation, specific to both the biological agent and the exact circumstances. Once ROM is lifted, an extended period of targeted health surveillance is necessary to ensure that any subsequent outbreak or re-emergence of disease is quickly detected.

ROM will have significant psychological impact on the main force, the personnel directly affected, the local civilian population and the perceptions of those at home. A decision to withhold information in order to reduce anxiety, although well intentioned, is likely to be counterproductive since rumour and confusion will spread quickly. It is important that all questions from the media are directed to Media Ops in accordance with local protocols.

Standard measures to investigate and control the disease episode will be implemented, requiring input from specialist and medical NBC staff. A separate epidemiological team may be required for each area of ROM and may not be recoverable until ROM exit criteria are met. Each area of ROM also requires stand-alone life support / logistics with appropriate methods for delivering food, water, medical care and for carrying out waste disposal (including clinical waste). The zoonotic potential of the biological agent should be considered in the implementation of appropriate control measures for animal populations (see chapter Animal Surveillance in NBC Defence Operations).

\section{References}

A. STANAG 2278: Medical Advice to Commanders on Restriction of Movement of BW Exposed Troops.

B. STANAG 3204: Aeromedical Evacuation (Annex $\mathrm{G}$ - Aeromedical Evacuation of BC contaminated casualties). 